\title{
HAMILTONICITY AND GENERALISED TOTAL COLOURINGS OF PLANAR GRAPHS
}

\author{
MieczysŁaW BorowiEcki \\ Faculty of Mathematics, Computer Science and Econometrics \\ University of Zielona Góra \\ e-mail: M.Borowiecki@wmie.uz.zgora.pl \\ AND \\ IZAK BROERE $^{1}$ \\ Department of Mathematics and Applied Mathematics \\ University of Pretoria \\ e-mail: izak.broere@up.ac.za \\ Dedicated to the memory of our friend, Peter Mihók
}

\begin{abstract}
The total generalised colourings considered in this paper are colourings of graphs such that the vertices and edges of the graph which receive the same colour induce subgraphs from two prescribed hereditary graph properties while incident elements receive different colours. The associated total chromatic number is the least number of colours with which this is possible.

We study such colourings for sets of planar graphs and determine, in particular, upper bounds for these chromatic numbers for proper colourings of the vertices while the monochromatic edge sets are allowed to be forests. We also prove that if an even planar triangulation has a Hamilton cycle $H$ for which there is no cycle among the edges inside $H$, then such a graph needs at most four colours for a total colouring as described above.
\end{abstract}

The paper is concluded with some conjectures and open problems.

Keywords: even planar triangulation, total colouring, Hamilton cycle, hereditary property.

2010 Mathematics Subject Classification: 05C15.

\footnotetext{
${ }^{1}$ This author is thankful to the University of Zielona Góra whose hospitality he enjoyed during the preparation of this paper; he is also supported in part by the National Research Foundation of South Africa (Grant Numbers 90841, 91128).
} 


\section{INTRODUCTION}

All graphs considered will be finite, undirected and simple. An additive hereditary graph property is a class of graphs which is closed under unions, subgraphs and isomorphisms. For undefined notions on graphs in general we refer the reader to [6], and for hereditary graph properties to [3].

We need a few well-known properties of graphs and list them with their standard names:

$\mathcal{O}=\{G \in \mathcal{I}: G$ is edgeless, i.e., $E(G)=\emptyset\}$,

$\mathcal{O}_{k}=\{G \in \mathcal{I}$ : each component of $G$ has at most $k+1$ vertices $\}$,

$\mathcal{D}_{k}=\{G \in \mathcal{I}: G$ is $k$-degenerate, i.e., the minimum degree $\delta(H) \leq k$ for each $H \subseteq G\}$,

$\mathcal{T}_{k}=\left\{G \in \mathcal{I}: G\right.$ contains no subgraph homeomorphic to $K_{k+2}$ or $\left.K_{\left\lfloor\frac{k+3}{2}\right\rfloor,\left\lceil\frac{k+3}{2}\right\rceil}\right\}$.

\section{Generalised Total Colourings}

Let $\mathcal{P}$ and $\mathcal{Q}$ be additive hereditary graph properties and let $C=\{1, \ldots, k\}$. Then a function $c: V \cup E \rightarrow C$ is a $\operatorname{total}(\mathcal{P}, \mathcal{Q})$-colouring of $G$ if

(1) $G\left[\left\{c^{-1}(i)\right\} \cap V\right] \in \mathcal{P}$, for all $i \in C$,

(2) $G\left[\left\{c^{-1}(i)\right\} \cap E\right] \in \mathcal{Q}$, for all $i \in C$,

(3) if $e=v u$, then $c(v) \neq c(e) \neq c(u)$, i.e., incident elements are coloured differently.

The minimum number of colours needed in a total $(\mathcal{P}, \mathcal{Q})$-colouring of $G$ is called the total $(\mathcal{P}, \mathcal{Q})$-chromatic number and is denoted by $\chi_{\mathcal{P}, \mathcal{Q}}^{\prime \prime}(G)$. Clearly, when $\mathcal{P}=\mathcal{O}$ and $\mathcal{Q}=\mathcal{O}_{1}$, a total $(\mathcal{P}, \mathcal{Q})$-colouring of a graph $G$ is nothing but a total colouring of $G$ so that $\chi_{\mathcal{O}_{\mathcal{O}}}^{\prime \prime}(G)=\chi^{\prime \prime}(G)$.

An acyclic $k$-colouring of a graph $G$ is a proper vertex $k$-colouring of $G$ satisfying the additional requirement that the subgraph induced by the union of every pair of colour classes is acyclic. The minimum $k$ such that a graph $G$ has an acyclic $k$-colouring is called the acyclic chromatic number of $G$ and is denoted by $\chi_{a}(G)$.

There is a close link between acyclic colourings of a graph $G$ and its total $\left(\mathcal{O}, \mathcal{D}_{1}\right)$-chromatic number.

Theorem 1. If a graph $G$ has an acyclic $k$-colouring, then $G$ has a total $\left(\mathcal{O}, \mathcal{D}_{1}\right)$ colouring with $k$ colours when $k$ is odd and with $k+1$ colours when $k$ is even.

Proof. If $k$ is odd, we start by choosing for each colour $i, \frac{k-1}{2}$ pairs of colour classes in such a way that each edge of $G$ is in the acyclic subgraph of $G$ induced by two colour classes. These choices, which remind one of a near 1-factorisation 
of $K_{k}$, clearly determines a total $\left(\mathcal{O}, \mathcal{D}_{1}\right)$-colouring with $k$ colours. If $k$ is even a similar choice of $\frac{k}{2}$ pairs of colour classes, now reminding one of a 1-factorisation of $K_{k}$, can be used to describe the required colouring of the edges but an extra colour might be needed to colour the vertices.

This result will be useful in the next section. We remark that there is a more general result, with a similar proof, which we only state (without proof) as

Theorem 2. Let $G$ be vertex $\mathcal{P}$-colourable with $k$ colours such that the vertices of the union of any two colour classes $V_{i}, V_{j}$ induce a graph with a property $\mathcal{Q}$. Then $G$ has a total $(\mathcal{P}, \mathcal{Q})$-colouring with $k$ colours when $k$ is odd and with $k+1$ colours when $k$ is even.

\section{Results for Classes of Planar Graphs}

A number of results from the literature can be combined with Theorem 1 to give bounds for the total $\left(\mathcal{O}, \mathcal{D}_{1}\right)$-chromatic number of some classes of planar graphs. To start with, we have for the set $\mathcal{T}_{3}$ of all planar graphs Borodin's Theorem [1], with the corollary now following immediately from this result and Theorem 1.

Theorem 3. Every planar graph has an acyclic 5-colouring.

Corollary 1. If $G$ is a planar graph, then $\chi_{\mathcal{O}_{, \mathcal{D}_{1}}}^{\prime \prime}(G) \leq 5$.

Next we do the same for some known results for subclasses of $\mathcal{T}_{3}$. The first result is by Borodin, Kostochka and Woodall [2].

Theorem 4. Every planar graph $G$ with girth $g(G) \geq 7$ has an acyclic 3colouring.

Corollary 2. If $G$ is a planar graph with girth $g(G) \geq 7$, then $\chi_{\mathcal{O}, \mathcal{D}_{1}}^{\prime \prime}(G) \leq 3$.

The case of planar 3-colourable graphs will be treated in full in the next section.

For planar bipartite graphs we have the following theorem of Kostochka and Mel'nikov [9].

Theorem 5. If $G$ is a planar bipartite graph, then $\chi_{a}(G) \leq 5$ and the bound is sharp.

Applying Theorem 1 again to these graphs would give the result: If $G$ is a planar bipartite graph, then $\chi_{\mathcal{O}, \mathcal{D}_{1}}^{\prime \prime}(G) \leq 5$. However, it is relatively easy to prove a better bound for this class. 
Theorem 6. If $G$ is a planar bipartite graph, then $\chi_{\mathcal{O}_{, \mathcal{D}}}^{\prime \prime}(G) \leq 4$ and the bound is sharp.

Proof. We can use two colours for a proper colouring of the vertices (since $G$ is bipartite) and two other colours for the edges by the theorem of Ringel [12].

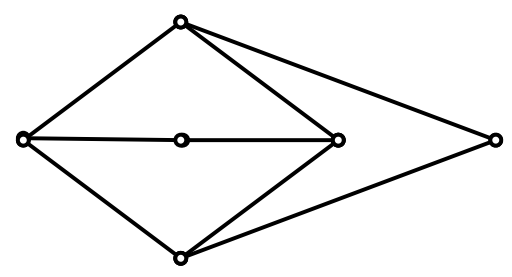

Figure 1

The bound is sharp since for the graph $G=K_{3,3}-e$ in Figure 1 we have $\chi_{\mathcal{O}, \mathcal{D}_{1}}^{\prime \prime}(G)=4$ : If there is a total $\left(\mathcal{O}, \mathcal{D}_{1}\right)$-colouring using only the three colours 1,2 and 3, then a contradiction can be derived in each of the following few cases.

(a) Only two colours, say 1 and 2, are used for the vertices of $G$-then each edge has to receive a colour different from 1 and 2 and two other colours are needed.

(b) All three colours are used for the vertices of $G$-then there are two nonadjacent vertices which have different colours. Each of the three cases in this situation are now easily checked to verify that a total colouring of $G$ using only these three colours cannot be completed.

Lastly we have, as a special case of the theorem of Ding et al. [7], the following theorem; using it in conjunction with Theorem 1 we immediately derive its corollary.

Theorem 7. Every $K_{5}$-minor free graph has an acyclic 5-colouring.

Corollary 3. If $G$ is a $K_{5}$-minor free graph, then $\chi_{\mathcal{O}, \mathcal{D}_{1}}^{\prime \prime}(G) \leq 5$.

\section{Hamiltonicity and Total Colourings of Even Triangulations}

We shall now continue the discussion of total $\left(\mathcal{O}, \mathcal{D}_{1}\right)$-colourings of classes of planar graphs by showing in Theorems 8 and 9 that many 3 -colourable planar graphs have total $\left(\mathcal{O}, \mathcal{D}_{1}\right)$-colouring with four colours such that only colours 1,2 and 3 are used to colour the vertices of the graph while colours 1,2, 3 and 4 are used to colour the edges of the graph. Since this idea was initially formulated by Peter Mihók as a conjecture for all 3-colourable planar graphs, we shall refer to such a total colouring as a Mihók colouring. 
In our results we often deal with plane embeddings of planar graphs and we find it convenient not to distinguish between such a graph and its embedding. It is useful to consider also the hamiltonicity of even planar triangulations; in this connection, we start right away with

Theorem 8. Every even planar triangulation which has a Hamilton cycle $H$ for which there is no cycle among the edges inside $H$ has a Mihók colouring.

Proof. Let $G$ be an even planar triangulation of order $n$ which has a Hamilton cycle $H$ for which there is no cycle among the the edges inside $H$. Using $H$, we identify three sets of edges of $G: E_{O}$, the set of edges outside $H, E_{H}$, the set of edges of $H$, and $E_{I}$, the set of edges inside $H$.

It is well known that $\chi(G)=3$ and we start by taking any 3-colouring of the vertices of $G$. (There is, in fact, up to a permutation of the colours used, a unique 3-colouring of the vertices of $G$.) Next we colour the edges using the same three colours: once the two colours of the end-vertices of an edge have been chosen as above, we colour the edge between them with the third colour. This is an $\left(\mathcal{O}, \mathcal{P}\right.$ )-total colouring of $G$ (with $\mathcal{P}=\mathcal{D}_{2}$ ) and will be referred to as the standard colouring.

Using the fact that there is no cycle among the edges in $E_{I}$, we now describe an $\left(\mathcal{O}, \mathcal{D}_{1}\right)$-total colouring of $G$ with four colours such that one of the colours is not used for any vertices of the graph but appears only at edges of the graph. This colouring is obtained by starting with the standard colouring of $G$ described above and recolouring each edge of $E_{I}$ with colour 4 . Since there is no cycle among these edges, it is immediate that the subgraph induced by the edges of colour 4 is in $\mathcal{D}_{1}$. It is also clear that each edge $u v$ receives a colour which differs from the colours of the vertices $u$ and $v$.

Finally, we need to prove that there is no cycle among the edges with colour $i$ for $i=1,2$ and 3 . In order to do this, we need only consider $G-E_{I}$. Note that, since $H$ is a Hamilton cycle, the subgraph $G-E_{I}$ of $G$ is a (maximal) outerplanar graph and the colours of the vertices and edges of this graph are those that were given to them in the standard colouring we started with.

Suppose now that there is a cycle $C$ in (any) one of these colours, say colour 1 . Then the vertices of $C$ (which are all on $H$ ) are coloured alternately 2 and 3 by the standard colouring. Hence the cycle $C$ is an even cycle. But the outside of $H$ is triangulated; hence $C$ has one or more diagonals (outside $H$ ) and one of them necessarily joins two vertices of $C$ which are both of colour 2 or both of colour 3 , a contradiction, since the standard colouring is a proper colouring on the vertices of $G$.

This proves that the subgraph induced by the edges of colour $i$ is also in $\mathcal{D}_{1}$ for $i=1,2$ and 3 and completes the proof. 
It is well known, as shown for example in [10], that every 3-colourable planar graph is a subgraph (although not necessarily an induced subgraph) of an even planar triangulation. The result of Theorem 8 therefore contributes to our belief in the following two conjectures.

Conjecture 1. $\chi_{\mathcal{O}^{\prime} \mathcal{D}_{1}}^{\prime \prime}(G) \leq 4$ for every 3-colourable planar graph.

Conjecture 2. Every 3-colourable planar graph has a Mihók colouring.

It is easy to see that these conjectures, if true, would be best possible too.

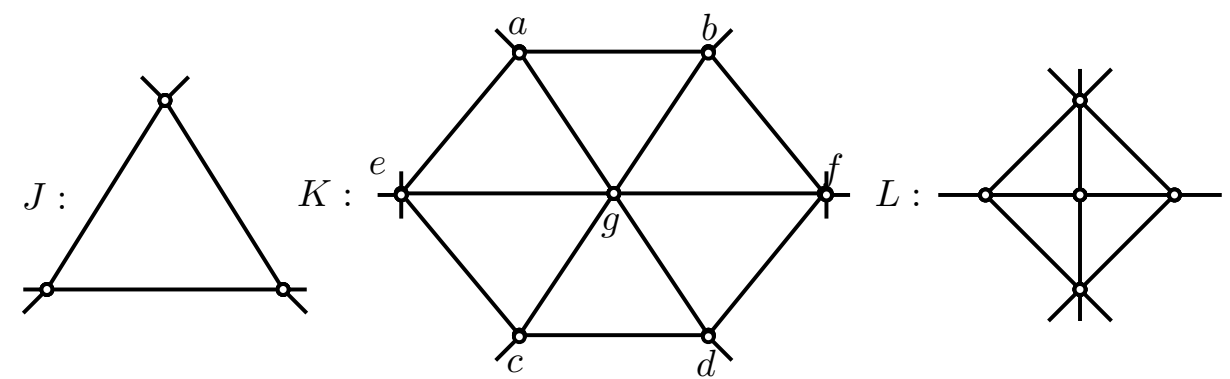

Figure 2

In the theorem which follows, we consider possible counterexamples to these conjectures. To formulate it, let $G$ be an even planar triangulation with minimum degree four which does not contain three vertices of degree four which are pairwise adjacent or which does not contain four vertices of degree four which are all adjacent to a vertex of degree six and induce two independent edges or it does not contain three vertices of degree four which are all adjacent to two vertices of degree greater than or equal to six and induce a path. We shall refer to these forbidden structures as three forbidden configurations of $G$; they are named $J$, $K$ and $L$, respectively, and depicted in Figure 2. Using three reductions, we are now ready to formulate and able to prove

Theorem 9. An even planar triangulation on the least number of vertices which does not have a Mihók colouring, if one exists, contains none of the forbidden configurations $J, K$ and $L$.

Proof. Suppose that the theorem is not true so that there is a counterexample, i.e., there is an even planar triangulation which does not have a Mihók colouring which contains at least one of these three forbidden configurations; yet every even planar triangulation with fewer vertices has a Mihók colouring. Let $G$ be such a counterexample. Then $G$ certainly contains no four pairwise adjacent vertices of degree four, for they would form a $K_{4}$ which is not a subgraph of $G$ since $G$ is 3 -colourable. We shall show below, under the headings Reduction 1, 2 and 3, 
that whichever one of the three configurations $J, K$ and $L$ are contained in $G$, it results in $G$ having a Mihók colouring.

Reduction 1 . If $G$ contains $J$, i.e., three pairwise adjacent vertices of degree four as depicted in Figure 3(a), then there will be three such vertices which are the only vertices in a face of an even planar triangulation with fewer vertices.

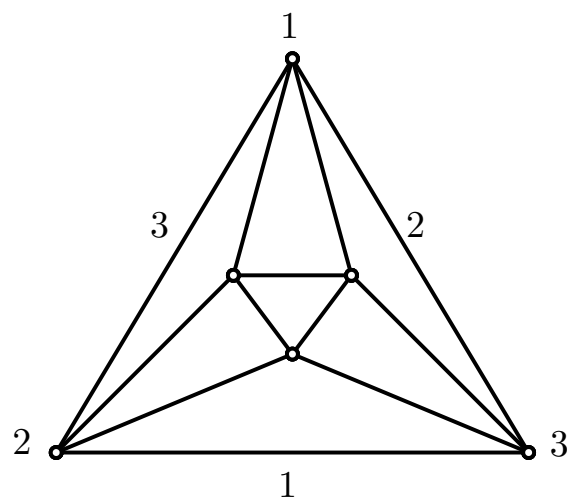

(a)

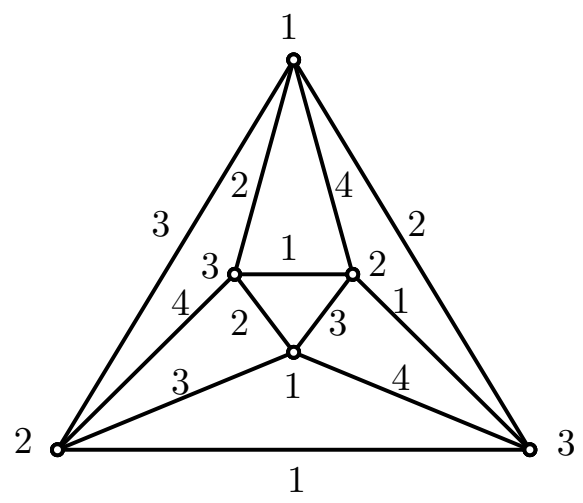

$(b)$

Figure 3

But this (smaller) even planar triangulation then has a Mihók colouring, which is also shown in Figure 3(a). In Figure 3(b) we now show how an extension of this Mihók colouring to a Mihók colouring of $G$ can be made. It could be remarked that this extension remains a Mihók colouring, even if one or two of the outside edges in Figure 3(a) have the colour 4.

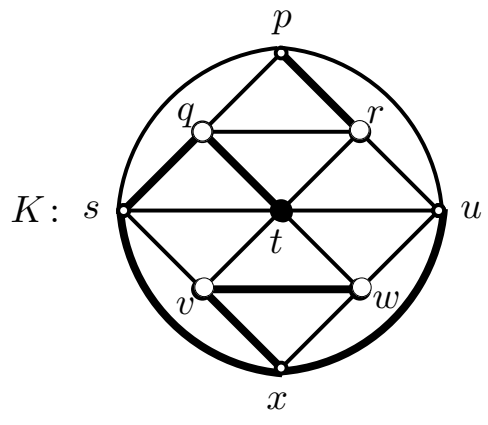

(a)

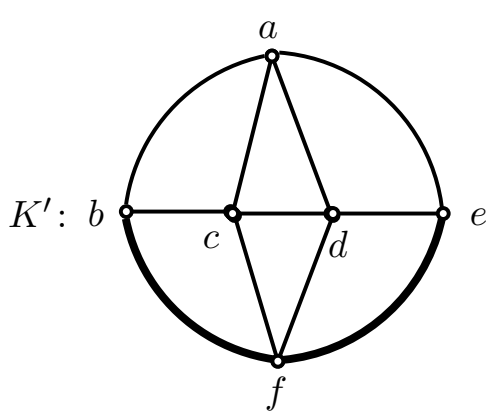

(b)

Figure 4

Reduction 2. Suppose $G$ contains $K$, i.e., four vertices of degree four which are all adjacent to a vertex of degree six and induce two independent edges. Then $G$ contains the configuration depicted in Figure 4(a), with $q, r, v$ and $w$ the vertices of degree 4 and $t$ the vertex of degree 6 . In this reduction we may of 
course assume that $p$ and $x$ are not of degree 4 , for otherwise we could employ Reduction 1 to deal with the case.

Again one can assume that there is a Mihók colouring of the smaller graph $G^{\prime}$ obtained by replacing $K$ in $G$ with the smaller configuration $K^{\prime}$ (depicted in Figure 4(b)). Consider any Mihók colouring of the graph $G^{\prime}$. Note that this colouring forces a unique colouring, up to a permutation of the three colours used, on the six vertices of $K^{\prime}$ and hence we may assume without loss of generality that this colouring assigns 1 to $a$ and $f, 2$ to $c$ and $e$ and 3 to $b$ and $d$. No matter how many and which of the four edges $p u, u x, s x$ and $p s$ are coloured with colour 4 and irrespective of the colours allocated to the other edges of $K^{\prime}$, the following colouring of $K$ completes a Mihók colouring of $K$ and thus of $G$.

Vertices: Colour $p, x, t$ with $1, u, q, v$ with 2 and $s, r, w$ with 3.

Edges: Use the same colours for $p u, u x, x s, s p$ as for $a e, e f, f b, b a$, respectively. Next colour $p r, r q, t u, t w, v x$ with 4 and give every other internal edge of $K$ the colour prescribed by the standard colouring. Since internal edges of $K$ induce no monochromatic cycle and there is no monochromatic path joining two vertices of $p, u, x, s$ containing internal edges, the resulting colouring is a Mihók colouring of $G$.

Reduction 3. Suppose $G$ contains $L$, i.e., it contains three vertices of degree four which are all adjacent to two vertices of degree greater than or equal to six and induce a path (see Figure 5(a) where names of such vertices are also indicated). A smaller graph $G^{\prime}$ is then obtained by contracting the vertices $r, s, t$ of the configuration $L$ to $e$ to obtain the smaller configuration $L^{\prime}$ and deleting parallel edges and loops (see Figure 5(b)). Clearly, $G^{\prime}$ is also an even planar triangulation.

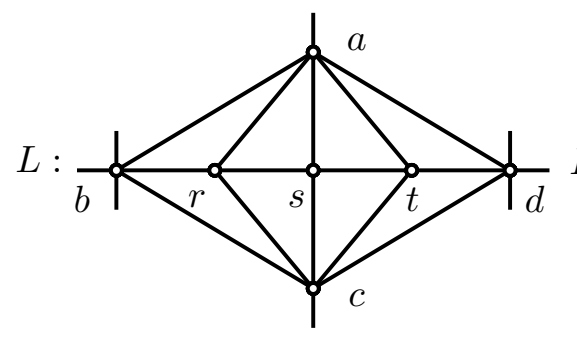

$(a)$

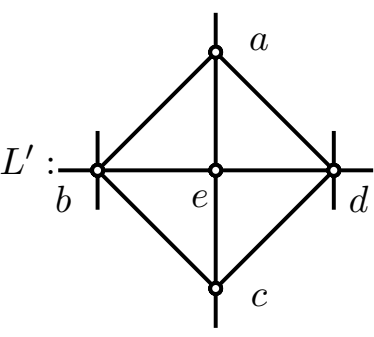

(b)

Figure 5

Consider any Mihók colouring of the graph $G^{\prime}$. Note that this colouring forces a unique colouring, up to a permutation of the three colours used, on the five vertices of $L^{\prime}$ and hence we may assume without loss of generality that this colouring assigns 1 to $e, 2$ to $b$ and $d$ and 3 to $a$ and $c$. We now describe partially how a Mihók colouring of $L$, and thus of $G$, can be obtained. 
Vertices: Use the same colours for $a, b, c$ and $d$ and colour $r$ and $t$ with 1 and $s$ with 2 .

Edges: Use the same colours for $a b, b c, c d$ and $a d$, and colour $c s$ with $1, r c, a t$ with 2 , st with 3 and as, ct with 4 .

Note that there should be at least one, but not all, of the four edges $a b, b c, c d$ and $a d$ that are coloured with colour 4 in the given colouring of $G^{\prime}$; hence there are, up to symmetry, four cases to consider on how colour 4 can be distributed among these four edges of $G^{\prime}$.

The proof can then be completed by considering, for each of these four cases, the subcases obtained by looking at all possible ways in which colours $1,2,3$, and 4 can be allocated to the remaining four edges inside $L^{\prime}$. These subcases are of course limited by the fact that only four edges of $L$ are uncoloured and that the given colouring of $G^{\prime}$ is a total $\left(\mathcal{O}, \mathcal{D}_{1}\right)$-colouring. These cases have been considered (and re-checked!) one-by-one by the authors but are very spaceconsuming to describe; hence they are left to the reader.

\section{Why the Graphs $J, K$ and $L$ Are Forbidden}

We now give a construction, for each of the three forbidden configurations, of an even planar triangulation which has no Hamilton cycle at all or which has a cycle among the edges inside and a cycle among the edges outside every Hamilton cycle. We start with the configuration $J$.

If an even planar triangulation does have three pairwise adjacent vertices of degree four, then it is possible that the graph has no Hamilton cycle. This can be seen by using the following construction: If $G$ and $H$ are planar triangulations and $\mathcal{F}$ is a set of faces of $H$, we construct the graph $H(\mathcal{F}, G)$ by putting a copy of $G$ in every face of $\mathcal{F}$ with the outside triangle of $G$ onto the triangle which is the boundary of the face in $\mathcal{F}$ under consideration. We shall refer to the graph $H$ in this construction as the big graph and to the copies of $G$ as the small graphs. Clearly, when $G$ and $H$ both are even planar triangulations, then so is $H(\mathcal{F}, G)$ for every choice of the set $\mathcal{F}$ of faces of $H$.

We shall now show that many graphs of the form $H(\mathcal{F}, F)$, with $H$ an even planar triangulation with at least four faces and $F$ the graph depicted in Figure 6, are non-Hamiltonian even planar triangulations. This can be seen by remarking that, if $H(\mathcal{F}, F)$ has a Hamilton cycle, it can only visit the three vertices inside a small copy of $F$ by entering the face of $H$ where this copy of $F$ is placed and exiting this face at two different (adjacent) vertices $u$ and $v$ of $H$ on this face. The function $\theta$, which assigns this edge $u v$ to every face in $\mathcal{F}$ is necessarily oneto-one: If two faces of $\mathcal{F}$ share such an edge, then the Hamilton cycle only visits the vertices on or in these two faces. 


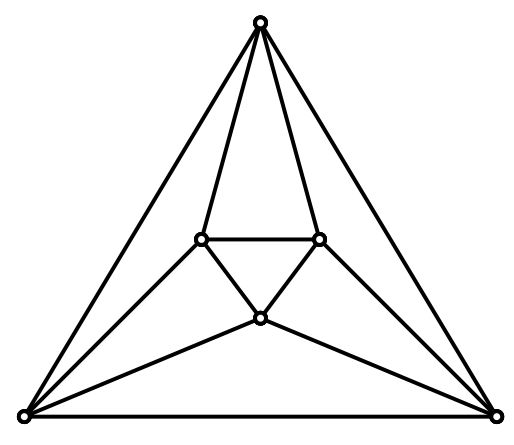

Figure 6

Hence $\theta(\mathcal{F})$ is a set $E$ of edges of $H$ with $|\theta(\mathcal{F})|=|E|$. Hence, if $H$ is a graph of order $n$ and $\mathcal{F}$ is chosen with $|\theta(\mathcal{F})|>n$, the edges of $\theta(\mathcal{F})$ will induce a subgraph of $H$ with at least one vertex of degree at least three. But then the Hamilton cycle of $H(\mathcal{F}, F)$ has a vertex of degree at least three, a contradiction. We remark that the choice above can, for example, be made in $F(\mathcal{F}, F)$ since $F$ has eight faces and six vertices.

The other forbidden configuration used in Theorem 9, depicted as the graph $K$ in Figure 2, is now used to create an even planar triangulation in which every Hamilton cycle has a cycle among its inside edges and one among its outside edges. This construction starts by taking a planar embedding of $K_{2, n}$ for an even $n \geq 6$; say with vertices $u$ and $v$, both of degree $n$ and $w_{1}, \ldots, w_{n}$, all of degree 2. The required graph is then obtained by inserting a copy of $K$ in each of the $n$ faces of this embedding and joining, for each of these copies, $a$ and $b$ to $u, c$ and $d$ to $v$ and identifying $e$ with $w_{i}$ and $f$ with $w_{i+1}$ (with arithmetic done modulo $n$ ).

Any Hamilton cycle of this graph will have to visit at least one copy of $K$ by traversing one of the paths eabgcdf or ecdgabf in this copy. However, both choices will leave two triangles; one with its edges on one side of $H$ and the other with its edges on the other side of $H$, see Figure 7 . In a similar way one can construct an even planar triangulation using $K_{2, n}$ and the forbidden configuration depicted as the graph $L$ in Figure 2, see Figure 7.

Yet another construction can be made from $K_{2, n}$ by using the graph in Figure 8 in a similar way as $K$ and $L$ are used (repeatedly) in Figure 7 . The graph obtained will then be an even planar triangulation in which the vertices of degree 4 induce an independent set so that the graph constructed does not contain any one of the three forbidden configurations $J, K$ and $L$. It is easy to prove that any Hamilton cycle $H$ of this graph will also not use the edges of (at least) two triangles; one with its edges on one side of $H$ and the other with its edges on the other side of $H$. 

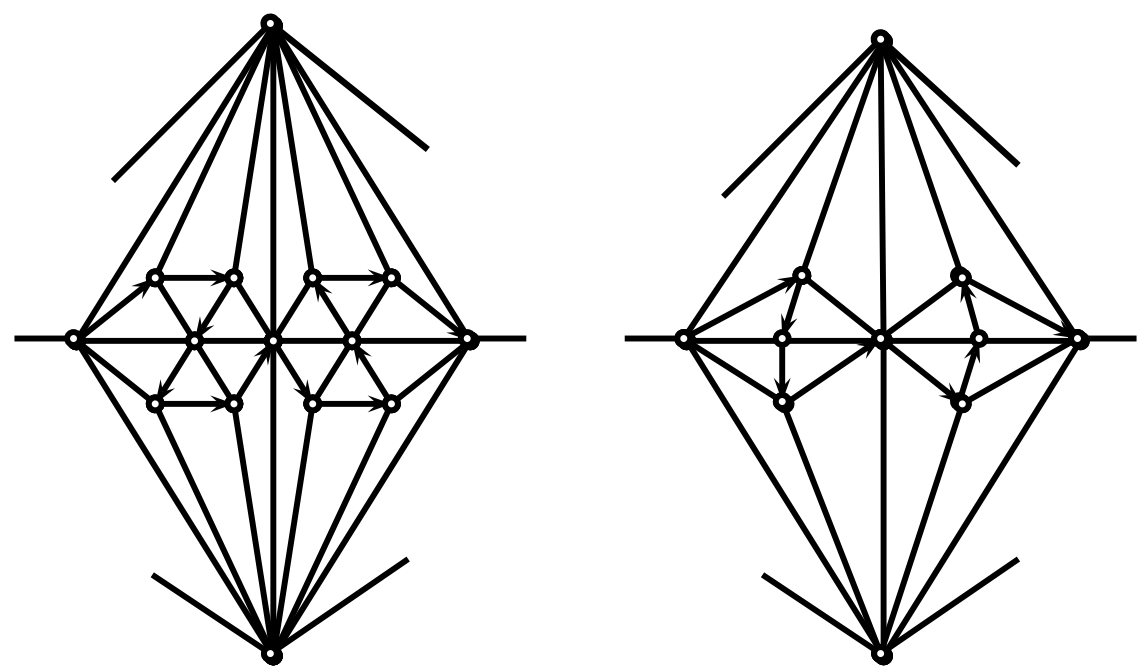

Figure 7

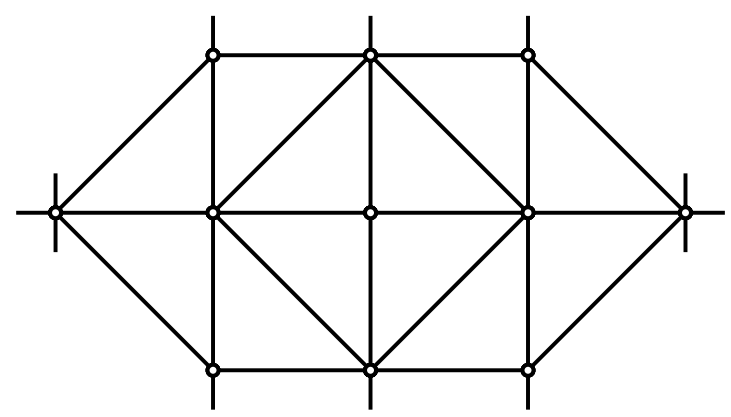

Figure 8

We remark that the set $\mathcal{F}=\{J, K, L\}$ of forbidden configurations is therefore not sufficient to prove a result which reads "If an even planar triangulation contains no graph in $\mathcal{F}$ as a forbidden configuration, then it has a Hamilton cycle $H$ for which there is no cycle among the edges inside $H$." This remark is proven by the last construction, since the graph constructed there does not contain any one these three forbidden configurations. It would be of interest to find a set $\mathcal{F}$ with sufficiently many graphs which would enable one to prove such a result.

\section{Minók-Type Total Colourings of a Class of Planar Graphs}

A graph $G$ is 1-immersed into a surface if it can be drawn on the surface so that each edge is crossed by at most one other edge. In particular, a graph is 1-planar if it is 1-immersed into the plane (i.e., has a plane 1-immersion). The notion of a 
1-planar graph was introduced by Ringel [11] in connection with the problem of the simultaneous colouring of vertices and faces of plane graphs in which adjacent vertices, adjacent faces and vertices incident to faces receive different colours.

A graph $G$ is outer-1-planar if $G$ can be 1-immersed into a plane such that all vertices are on the outer face. This notion was introduced by Eggleton [8]. It is clear that every outer-1-planar graph is planar.

An outer-1-planar graph $G$ is maximal if $G$ is not a proper spanning subgraph of an outer-1-planar graph. The characterization of maximal outer-1-planar graphs presented as Theorem 3.5 in [13] (where they are called pseudo-outerplanar graphs) is not quite correct. We give in Theorem 10 a corrected version (without proof). This characterization is constructive and starts with any maximal outer-1-planar graph $H$. To formulate it, we need two additional definitions.

Let $C$ be the boundary cycle of the outer face of a maximal outer-1-planar graph $G$. An edge $e \in E(G)$ is called external if $e$ belongs to the cycle $C$. An external edge $e$ is strong if $e$ belongs to a subgraph of $G$ isomorphic to $K_{4}$.

Theorem 10. Every maximal outer-1-planar graph $G$ is obtained from a maximal outer-1-planar graph $H$ by gluing a $K_{4}$ along an external edge of $H$ or by gluing $K_{3}$ along an external strong edge.

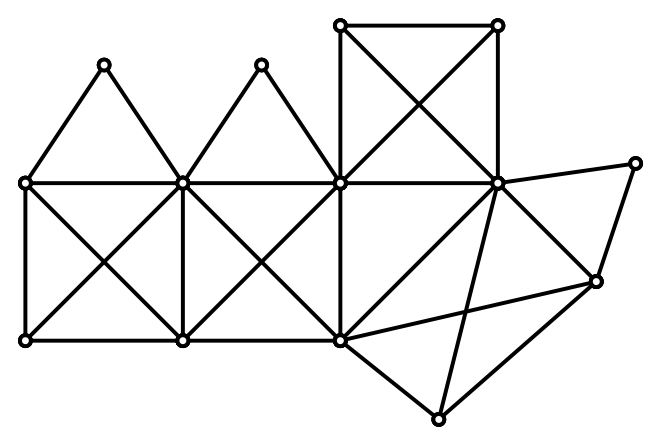

Figure 9

The example of a maximal outer-1-planar graph shown in Figure 9 can be obtained by choosing any triangle of this graph (which is the boundary of a face) for $H$, the maximal outer-1-planar graph with which the construction is started. Note that if we glue a $K_{3}$ to a $K_{3}$, the resulting graph is not a maximal outer-1planar graph. Hence if one starts the construction with $H=K_{3}$, the next step should be to glue a $K_{4}$; this also then creates the first strong edge.

Note also that it follows from Theorem 10 that every outer-1-planar graph is 3-degenerate.

Corollary 4. Let $G$ be a maximal outer-1-planar graph. Then there is a total $\left(\mathcal{O}, \mathcal{D}_{1}\right)$-colouring of $G$ with five colours such that 
- only colours 1,2,3 and 4 are used to colour the vertices of $G$ while

- colours 1,2,3,4 and 5 are used to colour the edges of $G$.

Proof. Let $G$ be a maximal outer-1-planar graph and consider a construction of $G$ as described in Theorem 10 using the graphs $H_{1}, H_{2}, \ldots, H_{p}$, in this order, with each $H_{i}$ isomorphic to $K_{3}$ or $K_{4}$.

Let $G_{1}:=H_{1}$, and let, for $i=1, \ldots, p-1$, the graph $G_{i+1}:=G_{i} \mid H_{i+1}$ be obtained by the operation of gluing $H_{i+1}$ to an edge of the graph $G_{i}$ so that $G_{p}=G$.

To colour the vertices of $G$ we apply the greedy algorithm to colour the vertices in the order in which they were added by the construction to form $G$. First colour properly the vertices of $G_{1}$ in an arbitrary order - this is easy since $G_{1}$ is isomorphic to $K_{3}$ or $K_{4}$. If all the vertices of $G_{i}$ have been coloured for some $i, 1 \leq i \leq p-1$, then the graph $G_{i+1}$ has one or two uncoloured vertices and they can also be coloured greedily in an arbitrary order. It now follows that $G$ is 4-colourable, and, if $K_{4} \leq G$, then $\chi(G)=4$.

Since $G$, when constructed in this way, has a "tree-like" structure, every two colour classes induce an acyclic graph. This property can also be deduced in an inductive way from the fact that, if $G_{i}$ has this property, then the graph $G_{i+1}$ also has it. Thus the acyclic chromatic number of $G$ satisfies $\chi_{a}(G)=4$. The result then follows by Theorem 1 and the proof is complete.

\section{CONClusion}

To conclude, we mention some open problems. Since for $K_{5}$-minor free graphs we have by [7] the same upper bound as for planar graphs one wonders if the Four Colour Theorem can be strengthened to

Conjecture 3. If $G$ is a planar graph, then $\chi_{\mathcal{O}, \mathcal{D}_{1}}^{\prime \prime}(G) \leq 4$.

This conjecture, which suggests a stronger result than the Four Colour Theorem, would clearly be very difficult to prove. A weaker conjecture is stated in [4].

Conjecture 4. If $G$ is a planar graph, then $\chi_{\mathcal{D}_{1}, \mathcal{D}_{1}}^{\prime \prime}(G) \leq 4$.

It seems not unreasonable to expect that a Mihók type $\left(\mathcal{D}_{1}, \mathcal{D}_{1}\right)$-total colouring might be used to prove this conjecture. By this we mean that the colouring of the vertices of a planar graph can perhaps be done using only the three colours 1,2 and 3 (which, independently of what we aim for in a total colouring, can be done since the arboricity of every planar graph is at most three by [5]) while the colours 1, 2, 3 and 4 are then used for the edges of the graph - similar to what was done in the proof of Theorem 8 . 
As has been mentioned in Corollary $1, \chi_{\mathcal{O}_{,} \mathcal{D}_{1}}^{\prime \prime}(G) \leq 5$ for any planar graph. Taking Corollary 4 in to account, we expect that a Mihók type $\left(\mathcal{O}, \mathcal{D}_{1}\right)$-total colouring might be useful to prove a stronger result.

Conjecture 5. If $G$ is a planar graph, then $G$ has $\left(\mathcal{O}, \mathcal{D}_{1}\right)$-total $(4,5)$-colouring, i.e., only colours $\{1,2,3,4\}$ are used to colour the vertices of a graph, and colours $\{1,2,3,4,5\}$ are used to colour the edges of the graph.

\section{Acknowledgement}

The authors wish to thank Elżbieta Sidorowicz whose comments led to an improvement of this paper.

\section{REFERENCES}

[1] O.V. Borodin, On acyclic colorings of planar graphs, Discrete Math. 25 (1979) 211-236. doi:10.1016/0012-365X(79)90077-3

[2] O.V. Borodin, A.V. Kostochka and D.R. Woodall, Acyclic colourings of planar graphs with large girth, J. Lond. Math. Soc. 60 (1999) 344-352. doi:10.1112/S0024610799007942

[3] M. Borowiecki, I. Broere, M. Frick, P. Mihók and G. Semanišin, A survey of hereditary properties of graphs, Discuss. Math. Graph Theory 17 (1997) 5-50. doi:10.7151/dmgt.1037

[4] M. Borowiecki, A. Kemnitz, M. Marangio and P. Mihók, Generalized total colorings of graphs, Discuss. Math. Graph Theory 31 (2011) 209-222. doi:10.7151/dmgt.1540

[5] G. Chartrand, H.V. Kronk and C.E. Wall, The point-arboricity of a graph, Israel J. Math. 6 (1968) 169-175. doi:10.1007/BF02760181

[6] R. Diestel, Graph Theory (Springer-Verlag, Berlin, 1997).

[7] G. Ding, B. Oporowski, D.P. Sanders and D. Vertigan, Surfaces, tree-width, cliqueminors, and partitions, J. Combin. Theory Ser. B 79 (2000) 221-246.

[8] R.B. Eggleton, Rectilinear drawings of graphs, Util. Math. 29 (1986) 149-172. doi:10.1006/jetb.2000.1962

[9] A.V. Kostochka and L.S. Mel'nikov, Note to the paper of Grünbaum on acyclic colorings, Discrete Math. 14 (1976) 403-406. doi:10.1016/0012-365X(76)90075-3

[10] M. Król, On a sufficient and necessary condition of 3-colorableness for the planar graphs I, Pr. Nauk. Inst. Mat. Fiz. Teoret. PWr. 6 (1972) 37-40.

[11] G. Ringel, Ein sechsfarbenproblem auf der Kugel, Abh. Math. Semin. Univ. Hambg. 29 (1965) 107-117. doi:10.1007/BF02996313 
[12] G. Ringel, Two trees in maximal planar bipartite graphs, J. Graph Theory 17 (1993) $755-758$.

doi:10.1002/jgt.3190170610

[13] X. Zhang, G. Liu and J.-L. Wu, Edge covering pseudo-outerplanar graphs with forests, Discrete Math. 312 (2012) 2788-2799. doi:10.1016/j.disc.2012.05.017

Received 18 June 2014

Revised 7 December 2015

Accepted 12 February 2016 